\title{
VYEPTI (eptinezumab-jjmr) Intravenous Drug as a Preventive Treatment of Migraine
}

Mani Vijayanandh

\begin{abstract}
Migraine occurs due to vasodilation of enzyme named calcitonin gene-related peptide (CGRP), and it binds to the receptors causing vasodilation. VYEPTI is a new intravenous drug for migraine and can be used as a preventive treatment. It is a monoclonal antibody that blocks CGRP binding to the receptor, and hence stopping vasodilation. Individuals with migraine will have to take this injection once in 3 months.

Keywords: Calcitonin gene-related peptide, Migraine, Monoclonal antibody, VYEPTI.

Pondicherry Journal of Nursing (2019): 10.5005/jp-journals-10084-12118
\end{abstract}

\section{INTRODUCTION}

"Migraine" is French by origin and originated from the Greek language/Greek "hemi crania" means "only half the head." In this world, migraine is the third most leading disease. ${ }^{1}$ Twenty percent of the world population are affected by migraine; and in South India, $25.2 \%$ suffer from migraine. Typically people will have repeated headache in a unilateral manner, nausea, irritant to sound, light, and last for 4-72 hours. Some individuals will have symptoms of neurological and autonomic dysfunction. ${ }^{2}$ Women are more affected than men. ${ }^{3}$ It also occurs due to psychiatric conditions such as phobia, depression, bipolar, panic, and risk of suicide. ${ }^{3}$ The study proved that patients showed better prognosis when treated of migraine and mental health problems. ${ }^{4}$

\section{Migraine: Overview}

Migraine is a kind of headache. Patients report one-side headache, which occurs due to a defect in brain activity. The triggering factors for migraines are changes in hormone levels, use of birth control pills, sleeping disturbance, alcohol intake, loud noises, photophobia, smoking, skipping meals, and stress. Migraine are of two types: one is classic (with aura) and another is common (without aura). Treatment of migraine consists of symptomatic management and lifestyle management. ${ }^{5-8}$

\section{Newer Approved Treatment of Migraine VYEPTI (Eptinezumab-jjmr)}

VYEPTI (eptinezumab-jjmr) is an intravenous drug used as a preventive treatment of migraine.

\section{What is the Medication?}

VYEPTI is an intravenous drug used as a preventive treatment of migraine in adults, and this drug is approved by the Food and Drug Administration.

\section{Clinical Pharmacology}

Mechanism of action-eptinezumab-jjmr is an antibody that adheres to the calcitonin gene-related peptide (CGRP) and blocks it to receptors and preventing vasodilation.
Department of Medical Surgical Nursing, Kasturba Gandhi Nursing College, Sri Balaji Vidyapeeth Deemed University, Puducherry, India

Corresponding Author: Mani Vijayanandh, Department of Medical Surgical Nursing, Kasturba Gandhi Nursing College, Sri Balaji Vidyapeeth Deemed University, Puducherry, India, Phone: +91 9524244889, e-mail: vanandh2010@gmail.com

How to cite this article: Vijayanandh M. VYEPTI (eptinezumab-jjmr) Intravenous Drug as a Preventive Treatment of Migraine. Pon J Nurs 2019;12(2):56-57.

Source of support: Nil

Conflict of interest: None

Pharmacodynamics - eptinezumab-jjmr does not have any clinical effects.

Pharmacokinetics-eptinezumab-jjmr exhibits increase in plasma level after a single dose which is administered once in 3 months. The half-life period is 27 days.

\section{How IS IT Used?}

\section{Recommended Dosing}

- Dosage: $100 \mathrm{mg}$ or $300 \mathrm{mg}$ once in 3 months

\section{Dilution}

Dilute only in $100 \mathrm{~mL} 0.9 \%$ sodium chloride solution.

\section{Nature}

VYEPTI is of slightly pale or dark background, brownish-yellow in nature.

\section{Important Precautions and Interactions}

\section{Reactions}

Hypersensitivity reactions such as angioedema, urticaria, facial flushing, and rash.

() The Author(s). 2019 Open Access This article is distributed under the terms of the Creative Commons Attribution 4.0 International License (https://creativecommons. org/licenses/by-nc/4.0/), which permits unrestricted use, distribution, and non-commercial reproduction in any medium, provided you give appropriate credit to the original author(s) and the source, provide a link to the Creative Commons license, and indicate if changes were made. The Creative Commons Public Domain Dedication waiver (http://creativecommons.org/publicdomain/zero/1.0/) applies to the data made available in this article, unless otherwise stated. 


\section{Temperature Maintenance}

Kept at $2-8^{\circ} \mathrm{C}$ till it is used and should not be exposed to light. Do not freeze or shake.

\section{About the PROMISE Clinical Trial Program}

PROspective Multicentre Imaging Study for Evaluation study was done to understand the effectiveness of VYEPTI ${ }^{\text {TM }}$ (eptinezumabjjmr). In this placebo-controlled study, two randomized groups were selected. The first group was PROMISE, which consisted of one patient with migraine for 4 days, one patient with chronic migraine, and other with 14 days of headache. The second group PROMISE consisted of two patients with 8 days of migraine and other with 26 days of headache. Patients were randomized and given 100 or $300 \mathrm{mg}$ of VYEPTI. ${ }^{9}$

Group I: the group received placebo $(n=222), 100 \mathrm{mg}(221)$, and $300 \mathrm{mg}$ (222) once in 3 months for 1 year. The patient with headache were classified into control and experiment group. The control group were in placebo effect, experiment group were given with $100 \mathrm{mg}$ and $300 \mathrm{mg}$ of VYEPTI drug the value were statistically analyzed based on that $100 \mathrm{mg} \mathrm{49.8 \%} \mathrm{(} p=0.009), 300 \mathrm{mg} \mathrm{56.3 \%}$ $(p<0.001)$ and $37.4 \%$ for placebo. Based on the findings, in the first 7 days of every month it is found to be that people in experiment group had migraine in lesser duration when compared with people were in control group. ${ }^{9}$

Group II: the group received placebo $(n=366), 100 \mathrm{mg}(356)$, and $300 \mathrm{mg}$ (350) once in 3 months for 6 months. The patient with headache were classified into control and experiment group. The control group were in placebo effect, experiment group were given with $100 \mathrm{mg}$ and $300 \mathrm{mg}$ of VYEPTI drug the value were statistically analyzed based on that $100 \mathrm{mg} \mathrm{57.6 \%}(p<0.001)$, $300 \mathrm{mg} \mathrm{61.4 \%}(p<0.001)$ and $39.3 \%$ for placebo. The patient in control group gets migraine in the first 7 days of a month when compared with patient in experiment group. ${ }^{9}$

\section{Conclusion}

Based on the above study, it is understood that the new drug VYEPTI (eptinezumab-jjmr) is effective when compared with the placebo-treated patients.

\section{References}

1. Ramasamy B, Karri M, Venkat S, Andhuvan G. Clinical profile and triggers of migraine. Int J Res Med Sci 2019;7(4):1050-1054. DOI: 10.18203/2320-6012.ijrms20191083.

2. Classification and diagnostic criteria for headache disorders, cranial neuralgias and facial pain. Headache Classification Committee of the International Headache Society. Cephalalgia 1988;8(Suppl 7): $1-96$.

3. Lipton RB, Hamelsky SW, Kolodner KB, Steiner TJ, Stewart WF. Migraine, quality of life, and depression: a population-based case-control study. Neurology 2000;55(5):629-635. DOI: 10.1212/ WNL.55.5.629.

4. Jayalakshmi S, Vooturi S. Migraine and mood disorders: prevalence, clinical correlations, and disability. J Neurosci Rural Pract 2019;10(1): 1-2. DOI: 10.4103/jnrp.jnrp_323_18.

5. Becker WJ. Acute migraine treatment in adults. Headache 2015;55(6):778-793. DOI: 10.1111/head.12550.

6. Diener H-C, Kurth T. Migraine and stroke. In: Grotta JC, Albers GW, Broderick JP, et al. Stroke: Pathophysiology, Diagnosis, and Management. 6th ed., ch. 43, Philadelphia, PA: Elsevier; 2016.

7. Marmura MJ, Silberstein SD, Schwedt TJ. The acute treatment of migraine in adults: the American Headache Society evidence assessment of migraine pharmacotherapies. Headache 2015;55(1): 3-20. DOI: 10.1111/head.12499.

8. Silberstein SD. Headache management. In: Benzon HT, Rathmell $\mathrm{JP}, \mathrm{Wu} \mathrm{CL}$, et al. Practical Management of Pain. 5th ed., ch. 30, Philadelphia, PA: Elsevier Mosby; 2014.

9. drugs.com.denmark: FDA Approves; Feb 2020. Available from https:// www.drugs.com/newdrugs/fda-approves-vyepti-eptinezumab-jjmrintravenous-preventive-migraine-5166.html. 